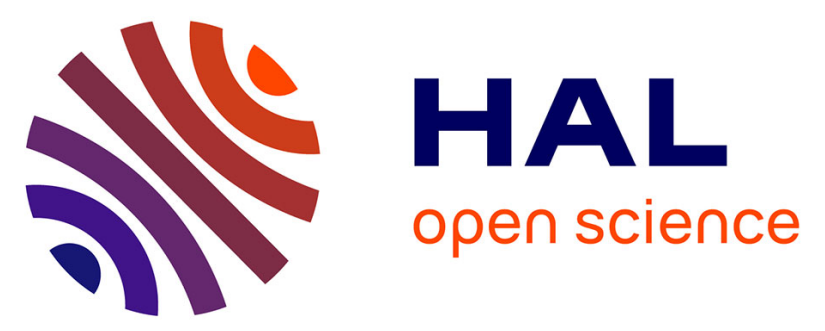

\title{
Machine Layout Design Problem Under Product Family Evolution in Reconfigurable Manufacturing Environment: A Two-Phase Based-AMOSA Approach
}

Hichem Haddou Benderbal, Lyes Benyoucef

\section{- To cite this version:}

Hichem Haddou Benderbal, Lyes Benyoucef. Machine Layout Design Problem Under Product Family Evolution in Reconfigurable Manufacturing Environment: A Two-Phase Based-AMOSA Approach. International Journal of Advanced Manufacturing Technology, 2019, 104 (1-4), pp.375-389. 10.1007/s00170-019-03865-1 . hal-02128566

\section{HAL Id: hal-02128566 \\ https://hal-amu.archives-ouvertes.fr/hal-02128566}

Submitted on 22 May 2020

HAL is a multi-disciplinary open access archive for the deposit and dissemination of scientific research documents, whether they are published or not. The documents may come from teaching and research institutions in France or abroad, or from public or private research centers.
L'archive ouverte pluridisciplinaire HAL, est destinée au dépôt et à la diffusion de documents scientifiques de niveau recherche, publiés ou non, émanant des établissements d'enseignement et de recherche français ou étrangers, des laboratoires publics ou privés. 


\title{
Machine Layout Design Problem Under Product Family Evolution in Reconfigurable Manufacturing Environment: A Two-Phase Based-AMOSA Approach
}

\author{
Hichem Haddou Benderbal and Lyes Benyoucef \\ Aix-Marseille University, University of Toulon, CNRS, LIS, Marseille, France \\ \{hicham.haddou-benderbal, lyes.benyoucef\}@lis-lab
}

\begin{abstract}
Reconfigurable manufacturing systems (RMSs) are designed to manufacture a specific product, incorporating the scalability to other products in the same family. This ability is based primarily on the reconfiguration capabilities offered by reconfigurable machines tools (RMTs). This paper addresses one of the most important aspects related to the reactivity of RMSs. More specifically, it considers the relations, which link the conceived system with two important environments: its logical environment, i.e., the product family (products that share similarities) in which the RMS can evolve, and its physical environment, i.e., the physical workshop that implements this RMS. We study the machine layout problem by considering the product family evolution where two sub-problems are addressed. The first sub-problem concerns the evolution of the product, in the same family, towards new products to meet the evolutions and the requirements of the customers. The second sub-problem deals with the machine layout problem based on the results of the first sub-problem. For this, our two-phase based approach combines the wellknown metaheuristic, archived multi-objective simulated annealing (AMOSA), with an exhaustive search based heuristic to determine the best machine layout for all the selected machines of the product family. The developed approach is based on the initially generated process plans of products (in the product family) for the RMS design under performance metrics. Moreover, the proposed layout must at its best, respects both the constraints imposed by the generated process plans and those depicting the available location in the shop floor where machines can be placed. An illustrative numerical example is proposed to demonstrate the applicability of our approach.
\end{abstract}

Keywords: Reconfigurable manufacturing system (RMS); changeable manufacturing system; layout; machine layout design; machine importance index; performance metrics; AMOSA.

\section{Context and motivations}

In an increasingly unstable political, economic, environmental and societal environments and under the pressure of globalization, growing competition, increasingly complex manufacturing systems, services, products and technologies with very short lifetimes, many companies are looking to rethink their produc- 
tion practices and philosophies. In fact, future productions in increasingly complex environments, customized, flexible and high-quality production look promising. So, to meet these requirements, companies need not only well-designed products, but also well-designed production systems. Moreover, the modern manufacturing environment is facing a paradigm shift that requires more changeability at both physical and logical levels. In this context, manufacturing systems must integrate this changeability. One of the major enablers of changeability is reconfigurability. From the perspective of industry 4.0, reconfigurability is essential to effectively adapt to the ever-increasing complexity of manufacturing environments. It allows rapid, efficient and easy adaptation of these systems while being proactive, timely and economically feasible. The objective is to respond to new internal and external constraints in terms of globalization, variety of products, mass customization, shorter lead times...

The reconfigurable manufacturing system (RMS) is a recent manufacturing paradigm driven by the high levels of responsiveness and efficiency (Koren et al., 2010). The high responsiveness and performance efficiencies make it a convenient manufacturing paradigm and flexible enabler of mass customization to face the complexity of manufacturing environments. This is possible, thanks to reconfigurable machine tool (RMT) which gives the RMS its customized flexibility and variety of alternatives. Moreover, reconfigurability is a non-functional requirement of the system, linked to its long-term behavior (Andersen, 2017). This implies that conventional approaches that consider only the immediate requirements of the system will not necessarily lead to dynamically changeable systems. Thus, it is necessary to design systems with a dynamic capacity for change and include the key factors of reconfigurability through adapting design approaches and/or developing new design methodologies. Hence, the goal is to design responsive systems based on reconfigurability features key.

This research work addresses the reconfigurable manufacturing systems (RMSs) design problem. More specifically, it considers the RMS design problem on the machine level and their interactions, while considering the product family evolution (Koren et al., 2018a). This is done through studying the relation that links the conceived RMS to its two environments respectively logical (i.e., the product family in which this RMS can evolve) and physical (i.e., the physical workshop implementing this RMS) that are treated as two sub-problems.

Linked to the logical environment, the first sub-problem concerns the evolution of the product, in the same family, towards new products to meet the evolutions and the requirements of the customers. Three criteria are optimized respectively: (1) the minimization of the evolution effort of the system during the transition within the product family, (2) the maximization of the average use of the machines in order to reduce at best the imbalance of the loads and (3) the maximization of the presence of replacement machines in the production lines of the product family. While, linked to the physical environment, the sec- 
ond sub-problem is related to the machine layout. It is based on the results of the first sub-problem. Indeed, the physical structure of the workshop must be able to guarantee the necessary flexibility to follow the evolutions of the RMS in an efficient and fast way.

For this, a two-phase based approach combing the well-known metaheuristic, archived multi-objective simulated annealing (AMOSA), with an exhaustive search based heuristic is developed in this paper. It aims to guarantee the best transition between the different products of the same family via the different manufacturing ranges (first sub-problem) by determining the best layout of the selected RMTs (second sub-problem). Moreover, to better guide the final RMTs that integrate our RMS design, we adapt the machine importance index proposed by Haddou Benderbal et al. (2018a), which integrate the product family evolution. This later quantifies the importance of selected machines within each process plan of the product family and helps to ensure a better responsiveness and a high performance of the designed system while considering the changes imposed by the product family.

The rest of the paper is organized as follows: Section 2 summarizes the literature review dedicated to the RMS design and the machine layout design problems. Section 3 discuses the problem under consideration and its mathematical formulation. Section 4 presents the developed two-phase based-AMOSA approach. Section 5 shows an illustrative numerical example. Section 6 concludes the paper with some future research directions.

\section{Literature review}

Considered as one of the latest manufacturing paradigms, the RMS design, implementation, layout optimization, reconfigurable control, as well as process planning and production scheduling are belong the most active research topics in this field (Bortolini et al., 2018, Koren et al., 2018a, Maganha et al., 2018, Andersen et al., 2015, Renzi et al., 2014, Rehman and Babu, 2013, Bi et al., 2008). However, in this section, we will restrict and summarize the most recent research works dealing with respectively RMS design, RMS and process planning, RMS and product family, and RMS and layout.

\subsection{RMS design}

To design a RMS, two main steps are necessary (Koren et al., 2018a). From one hand, the first step concerns the process plans generation to determine the set of machines that integrate the RMS design. From another hand, the second step determines how this set of machines will be arranged/rearranged using candidate locations of the shop floor through layout design process. Koren et al. (2018a) formulated the design and operational principles for RMSs. According to these principles, they provided a state-of-the-art review of the design and operations methodologies of RMSs and discussed the role of intelligent manufacturing technologies in enhancing RMS performance, and how recent development of advanced diag- 
nostics and cyber physical manufacturing systems can facilitate the design and operations of RMS. Finally, they claimed that "future research should be conducted on the concurrent design of the productsystem-business strategies utilizing the RMS concept and principles, as well as for more effective methodologies for real-time RMS operations, for both machining and assembly systems".

Koren and Shpitalni (2010) presented the core characteristics and design principles of RMSs, and described the structure recommended for practical RMS with RMS core characteristics. A rigorous mathematical method is introduced for designing a RMS with this recommended structure. Dou et al. (2010) developed a GA-based approach for optimizing multi-part flow-line (MPFL) configurations of RMS for a part family. The objective is to minimize the capital cost of MPFL configurations. Using a case study, they demonstrated the efficiency of the proposed GA-base approach comparing to a particle swarm optimization algorithm. Huang et al. (2011) considered the problem of configuration selection in RMSs. They proposed a modified simulated annealing (SA) approach for managing the configuration selection and optimizing an economic objective function that includes the reconfiguration cost and the fixed cost in the given reconfiguration period. Furthermore, Saxena and Jain (2012) developed a configuration design three-phase methodology for RMSs where the goal is to optimize an economic objective function including capital cost of machine investment, reconfiguration cost, operating cost, maintenance cost and residual marginal value over time. Phase 1 aims at RMS evolution scenario and the mathematical modelling. Phase 2 evolves RMS configuration alternatives. Phase 3 aims at RMS configuration alternative optimization using mathematical model and artificial immune system.

Bensmaine et al. (2013) addressed the problem of RMSs design based on products specifications and reconfigurable machines capabilities. The problem concerns the selection of candidate reconfigurable machines among an available set, which will be then used to carry out a certain product based on the product characteristics. The selection of the machines considers two main objectives respectively the minimization of the total cost (production cost, reconfiguration cost, tool changing cost and tool using cost) and the total completion time. An adapted version of the non-dominated sorting genetic algorithm (NSGA-II) is proposed to solve the problem.

Wang and Koren (2013) discussed the scalability issues of incrementally changing production capacity of RMSs by adding or removing machines. They developed a new approach based on genetic algorithm for scalability planning that simultaneously changes the system configuration and rebalances the reconfigured system. In the same context, Koren et al. (2017) proposed a mathematical method maximizing the system throughput after reconfiguration and validated it using a real life case study. 
Battaïa et al. (2017) developed a decision support approach for the design of reconfigurable rotary machining systems with turrets used for producing several families of parts. They used a mixed integer program to model the complex design constraints such as compatibility and productivity requirements as well as design objectives. The model allows taking efficient decisions about part orientations, selection of machining modules and configuration/reconfiguration of working positions depending on the part families to be produced.

Recently, Koren et al. (2018b) compared typical configurations of large-volume manufacturing systems for mechanical products from cost, responsiveness and product quality perspectives. Comparing to traditional serial lines and pure parallel systems, they discussed two practical configurations - parallel serial lines, and RMSs. Haddou Benderbal et al. (2018ab) argued the necessity to integrate performance metrics at the outset of the design phase of RMS in order to enhance the performance of this later. Moreover, Gaddala and Xue (2018) addressed the problem of optimal configurations and reconfiguration processes for design of reconfigurable machine tools. A generic design AND-OR tree is used to model different design solution candidates, their machine configurations and parameters of these configurations. Where, a multi-level and multi-objective hybrid approach is developed to obtain the optimal design. To illustrate the approach, a case study is presented and the numerical results analysed.

\subsection{RMS and process planning}

When considering process plan generation in RMS, the state of the art is rich. Nallakumarasamy (2011) defined the process plan as 'the activity that decides the sequence, which the manufacturing process must follow'. This activity defines the operations order required to complete a single unit of product. It assigns each operation to the appropriate machine under the adequate configuration. ElMaraghy (2007) claimed that "we need to associate the evolutions, reconfigurations and reconfigurable process plans to changes and evolutions of manufacturing systems and products". Moreover, Shabaka and ElMaraghy (2007) developed a new genetic algorithm based model to perform process plan manufacturing costs in RMS environment. The model simultaneously considers all process plan parameters such as machine assignment and machine configuration.

Musharavati and Hamouda (2012ab) investigated the use of simulated-annealing-based algorithms in solving process planning problem for a RMS. They developed several variants of the simulated annealing algorithms respectively a variant of the basic simulated annealing algorithm, a variant of the simulated annealing algorithm coupled with auxiliary knowledge and a variant of the SA algorithm implemented in a quasi-parallel architecture. The obtained experimental results showed the superiority of the variants in comparison to a basic simulated annealing algorithm. Maniraj et al. (2014) proposed a two-phase-based 
ant colony optimisation approach to solve the process plan generation problem of a single product flowline in a reconfigurable context. In the first phase, priority-based encoding technique is applied to find feasible operation clusters. Where, in the second phase, ant colony technique is used for minimising the total cost of the RMS. A case study is presented to demonstrate the applicability of the developed approach.

In a multi-objective context, Chaube et al. (2012) and Bensmaine et al. (2013) proposed an evolutionarybased approach to solve the problem. Chaube et al. (2012) adapted the non-dominated sorting genetic algorithm (NSGA-II) where two objectives are considered, respectively, the total completion time and the total manufacturing cost. Bensmaine et al. (2013) integrated the process plan generation with the design problem using the same approach. Haddou Benderbal et al. (2017a) proposed a new flexibility metric to generate efficient process plan by integrating unavailability constraints of the selected machines. The resulting multi-objective problem is solved using an adapted version of NSGA-II.

More recently, Touzout and Benyoucef (2018a) tackled the sustainable process plan generation problem for a RMS, where the amount of greenhouse gases (GHG) emitted during the manufacturing process is minimized in addition to the total production cost and completion time. The authors developed an iterative multi-objective integer linear programming (I-MOILP) approach and compared with adapted versions of the archived multi-objective simulated-annealing approach (AMOSA) and the NSGA-II approach. They studded the influence of the probabilities of genetic operators on the convergence of the adapted NSGA-II and illustrated the applicability of the three approaches using numerical examples. Furthermore, inspired by (Touzout and Benyoucef, 2018a) and a microscopical study of the optimal Pareto front solutions generated by I-MOILP, Touzout and Benyoucef (2018b) developed three hybridmetaheuristics using an adapted version of AMOSA with 2-opt heuristic to solve the multi-objective multi-unit process plan generation problem for a RMS.

\subsection{RMS and product family}

It is well know that, "the design of RMS revolves around product family which can provide all the variety within a product family and can be subsequently reconfigured to accommodate future products belonging to distinct product families" (Ashraf and Hasan, 2016). Moreover, Bortolini et al. (2018) stated that "in a typical RMS, products are grouped in families and each of them requires a different system configuration. In this way, the system is configured to produce the first family and, once the production is finished, the system is reconfigured to produce the second family and so on", and 'product families are based on operational similarities among parts or on operation sequence similarity'. 
Following their state of the art, Bortolini et al. (2018) observed that few grouping methods are proposed and tested for RMSs, e.g. clustering techniques and MCDM methods as analytical hierarchical process (AHP) and analytical network process (ANP).

Since the effectiveness of a RMS depends on the formation of the best set of product families, Galan et al. (2007) presented a methodology for grouping products into families taking into account modularity, commonality, compatibility, reusability, and product demand as the most requirements of products in RMSs. For each product requirement, a matrix that summarises the similarity between pairs of products is first computed. Then, through the use of the AHP, a unique matrix that comprises the similarity values between products is obtained. Then, in order to obtain a dendogram that shows the diverse sets of product families that may be formed, the average linkage-clustering algorithm is applied to this matrix. Moreover, to cope with variations in capacity and functionality during product family formation, Pattanaik and $\mathrm{Ku}$ mar (2011) proposed a bi-criterion evolutionary genetic algorithm to identify the Pareto optimal solutions representing the product families for the RMS.

Goyal et al. (2013) proposed a novel operation sequence based bypassing moves and idle machines (BMIM) similarity coefficient to deal with the problem of part family formation in RMS. The operation sequence similarity based approach evaluates the differences among parts by measuring the number of idle machines and bypass movements considering fix operation sequences for the products. In the same context, Wang et al. (2016) presented a similarity coefficient approach for the formation of RMSs part family, which considers bypassing moves and idle machines. The approach considers both bypassing movements and idle machines with the goal of minimising idle machines and improving the smoothness of the part movements.

Kashkoush and ElMaraghy (2014) developed the first product family formation method that particularly addresses reconfigurable assembly systems (RASs). They used respectively product assembly sequences, product demand and commonality, as similarity coefficients. Then, hierarchical clustering is applied to generate various groups of product families that may be formed based on each similarity coefficient. Finally, they applied a novel consensus tree-based method to find the best aggregation for the three different hierarchical clustering trees.

\subsection{RMS and layout}

It is well known that machine layout influences the entire manufacturing system in terms of productivity and responsiveness. Thus, the machine layout problem is regarded as a facility layout problem (FLP) by several authors (Altuntas and Selim, 2012). Singh and Sharma (2006) and Drira et al. (2007) analyzed the existing literature dedicated to FLP through larger and non-restricted approaches. For conventional manu- 
facturing systems like flexible manufacturing systems (FMS), the literature identified five different configurations of machine layout—open field layout, loop layout, single row layout (known also as spin layout), multi-row layout (known also as ladder layout) and robot centered layout (Yang et al., 2005, Drira et al., 2007). Moreover, based on the chosen material handling system and possible flows allowed for products, many configurations could be determined for machine layout (Devise and Pierreval, 2000).

Heragu et al. (2001) presented a framework that determines the layout of manufacturing systems that are defined by recurrent product volumes and mix change. In the same context and to handle the next generation factory layouts design, Benjafaar et al. (2002) argued that there are two major approaches. First, by considering various manufacturing periods through the development of more robust layouts. Second, by developing flexible layouts. The authors stated that the reconfiguration of these layouts must be ensured by minimal effort in order to fulfill the frequent production requirement changes. Sharma and Singhal (2016) handled the layout design problem using a procedural approach based on an altered version of the traditional systematic layout planning (SLP).

Even though the layout is considered as one of the main steps in RMS design, Bortolini et al. (2018) claimed that "despite the first definition of RMS appears in 1999, the literature review shows that the investigation of a possible relationship between RMS and layout is fairly recent". In fact, we find a dearth of research works when it comes to the integration of layout problems with the design of RMS. Guan et al. (2012) developed a revised electromagnetism-like mechanism (REM) approach using automated guided vehicle (AGVs) as transportation systems for the layout design of RMSs. Where, the total material handling costs is the unique criterion to minimize. The obtained numerical results showed that the proposed REM is able to get optimal solutions for small-scale problems and near optimal solutions within limited computation time for large-scale problems.

To overcome the main limits existing in the literature as the consideration of a single objective in the definition of models addressing the layout design problem, Goyal et al. (2016) studied the design of reconfigurable flow lines (RFL) problem. They proposed a multi-objective optimization approach, where the cost, machine utilization, operational capability, and configuration convertibility are the conflicting objectives. An adapted version of the well-known multiple-objective particle swarm optimization (MOPSO) is implemented to handle the discrete and discontinuous solution search space. However, they didn't consider the layout design problem properly.

Azevedo et al. (2017) developed a multi-objective approach addressing the reconfigurable multi-facility layout problem. Three objectives are optimized respectively, the minimization of material handling costs, 
the maximization of the adjacency among departments and the minimization of the unsuitability of the department position and location (ie. departments inside facilities).

Recently, Haddou Benderbal et al. (2017b) proposed a metric to quantify the layout evolution effort considering product family in order to ensure the RMS high performance. An adapted version of AMOSA is proposed to solve the problem. Furthermore, Haddou Benderbal et al. (2018a) developed an exhaustive search based approach to resolve the machine layout problem while integrating the RMS design. This later didn't consider the influence of the product family on the machine layout.

To the best of our knowledge and from our literature review, little works have considered the machine layout problem for RMS design while considering the product evolution within the same family. In this research work, as a natural extension of the works presented in Haddou Benderbal et al. (2017b and 2018a), we try to tackle this complex problem.

\section{Problem description and formulation}

\subsection{Problem description}

Machine layout problem takes another dimension when it comes to RMS. In fact, in addition to the basic constraints related to the classical layout problem, it is necessary to integrate the characteristics related to RMS, and particularly those related to its reconfigurability. Moreover, the consideration of all these constraints and characteristics from the outset of the design phase of the workshop will ensure a better responsiveness and a very good level of performance (ability to reconfigure, flexibility, ...) of the designed RMS. This paper aims to develop a decision support approach to ensure the best transition between process plans of several products in the same product family, while maintaining the performance of each process plan.

From one hand, in order to increase the responsiveness of the system that faces frequent characteristic evolution of the product to be manufactured, it is necessary to design a RMS that offers capabilities to increase the number of alternative solutions within the generated process plans. Thus, our first challenge addresses the reaction to products changes. We propose an approach to facilitate existing resources sharing (selected RMTs) when integrating a new product from the product family. The aim is to foster the use of the same selected RMTs in process plans of different products of the same family. However, it is necessary to note that, the resource sharing is anticipated from the outset of RMS design (i.e., when selecting machines to manufacture the first product in the product family).

Our second challenge is to consider the best layout for these machines on the shop floor. Thus, this second challenge considers how selected machines - for each product in the product family — are going to 
be positioned and arranged on the shop floor. This positioning must maintain at its best, the high performance of the designed RMS as treated in the first challenge. Moreover, knowing that there is diverse layout configuration, the challenge is also to respect as best as possible the constraints imposed by the chosen layout configuration.

These two challenges are represented by two linked sub-problems. The first sub-problem deals with the logical environment linked to the designed RMS that treats the transition effort evolution between products from the same family. Whilst the second sub-problem covers the physical environment linked to the designed RMS and treats the machine layout problem.

Tackling the above two sub-problems will help us first minimizing the transition effort needed (the first sub-problem) when moving from one product to another within the same family while maintaining performances provided by the given process plans. It will also help reducing the number of constraints imposed by the selected machines as well as the shop floor on which they will be positioned (the second sub-problem). This is done by proposing a genuine tool to support decision-making for resources management (e.g., machine selection) to ensure the best transition between layouts of the selected machines involved in the RMS design for a product family.

Our literature review shows also the existence of various machine layout configurations for the shop floor. Thus, to make our model generic, we enable it to cover numerous types and possibilities for these layout configurations as detailed in sub-section 3.2.2.

\subsection{Problem formulation}

For a better understanding of the problem, Table 1 presents the used notations.

Table 1. Notations

\begin{tabular}{|c|c|}
\hline NP & Number of products in the product family \\
\hline NL & Number of total candidate locations on the shop floor \\
\hline NPM & $\begin{array}{l}\text { Number of candidate machine used to generate input process plans for each } \\
\text { product of the product family }\end{array}$ \\
\hline NSMp & Number of selected machines for the product $\mathrm{Pp}$ \\
\hline NM & Number of distinct selected machines for the product family \\
\hline $\mathbf{L}_{1}, \ldots, \mathbf{L}_{\mathrm{NL}}$ & Available locations \\
\hline $\mathbf{M}_{1}, \ldots, \mathbf{M}_{\mathrm{Nm}}$ & Available machines \\
\hline$P_{1}, \ldots, P_{N L}$ & Considered product from the product family \\
\hline $\mathrm{NC}_{\mathbf{j}}$ & Number of available configurations for the machine $M_{j}$ \\
\hline
\end{tabular}




\begin{tabular}{|c|c|}
\hline$C_{1}^{j}, C_{2}^{j}, \ldots, C_{N_{j}}^{j}$ & Configurations of the machine $\mathrm{M}_{\mathrm{j}}$ \\
\hline OPTNp & Total number of operations for the product $\mathrm{Pp}$ \\
\hline TFOP & Number of all operations of the product family \\
\hline $\mathbf{O P} \mathbf{P}_{1}^{\mathbf{p}}, \ldots, \mathbf{O P}_{\mathrm{OPTNp}}^{\mathrm{p}}$ & Operations of the product $\mathrm{Pp}$ \\
\hline $\mathrm{NO}_{\mathrm{p}}\left[\mathrm{M}_{\mathrm{j}}\right]$ & Number of occurrences of the machine $\mathrm{M}_{\mathrm{j}}$ for product $\mathrm{Pp}$ \\
\hline $\mathbf{C}_{\mathbf{l}}^{\mathbf{j}}(\mathbf{u})$ & The configuration 1 of the machine $\mathrm{M}_{\mathrm{j}}$ on which the operation $\mathrm{u}$ is realized \\
\hline $\mathbf{M}_{\mathbf{j}}\left(\mathbf{O P} \mathbf{P}_{\mathbf{u}}^{\mathbf{p}}\right)$ & The operation $u$ of the product $P p$ is being executed on the machine $M_{j}$ \\
\hline $\operatorname{Conf}_{\mathbf{j}}[]$ & Matrix of available configurations for the machine $M_{j}$ \\
\hline $\mathbf{P M}_{\mathbf{p}}\left[\mathbf{M}_{\mathbf{j}}\right]$ & Matrix of selected machines for product $p$ \\
\hline $\mathbf{M T}_{\mathbf{j}}[]$ & Matrix of available tools for the machine $\mathrm{M}_{\mathrm{j}}$ \\
\hline $\operatorname{MTOP}\left[O P_{u}^{p_{u}}\right]$ & Matrix of required tools for the operation $\mathrm{u}$ of the product $\mathrm{Pp}$ \\
\hline $\operatorname{PRM}\left[O P_{u}^{p}\right]\left[O P_{u}^{p}\right]$ & Operations precedence matrix \\
\hline $\operatorname{Min} A D\left[M_{j 101}\right]\left[M_{j 202}\right]$ & $\begin{array}{l}\text { Minimum accepted distance between the occurrence } \mathrm{o} 1 \text { of the machine } \mathrm{M}_{\mathrm{j}} 1 \text { and } \\
\text { the occurrence } \mathrm{o} 2 \text { of the machine } \mathrm{M}_{\mathrm{j}} 2 \text { (if } \mathrm{j} 1=\mathrm{j} 2 \text { then } \mathrm{o} 1 \neq \mathrm{o} 2 \text { ) }\end{array}$ \\
\hline $\operatorname{MaxAD}\left[\mathbf{M}_{\mathbf{j 1 0 1}}\right]\left[\mathbf{M}_{\mathbf{j 2 0 2}}\right]$ & $\begin{array}{l}\text { Maximum accepted distance between the occurrence of of the machine } \mathrm{j} 1 \text { and } \\
\text { the occurrence } \mathrm{o} 2 \text { of the machine } \mathrm{j} 2(\mathrm{if} \mathrm{j} 1=\mathrm{j} 2 \text { then } \mathrm{o} 1 \neq \mathrm{o} 2)\end{array}$ \\
\hline $\mathbf{P}\left[\mathbf{M}_{\mathbf{j o}}\right]$ & Position of occurrence of the machine $\mathrm{M}_{\mathrm{j}}$ \\
\hline
\end{tabular}

In this research work, the following assumptions are assumed:

Assumption 1: The set of machines involved in the RMS design is already selected. Thus, the initial optimal process plans of the different products, from the same family, are already generated based on performance metrics as proposed by Haddou Benderbal et al. (2017a, 2018b). Table 2 represents the used process plan structure.

Table 2. Process plan structure of product $\mathrm{P} 1$

\begin{tabular}{ccccccccc}
\hline Operation & OP2 & OP5 & OP1 & OP6 & OP7 & OP9 & OP3 & OP13 \\
\hline Designated machines & M2 & M10 & M1 & M10 & M4 & M6 & M1 & M3 \\
\hline
\end{tabular}

Assumption 2: For each product to be manufactured from the product family, a single unit of a product is considered.

Assumption 3: Each unit from the product family is assigned to its optimal process plan. Thus, the best set of selected machines to design the RMS and their respective occurrences are known. 
Assumption 4: The set of machines used to generate the process plans is also known and it is used for solving the first sub-problem (i.e. layout evolution effort).

Assumption 5: The layout configuration comprises a number $N L$ of distinct locations $L$. Each machine $\boldsymbol{M}_{\boldsymbol{j}}$ has a limited number of occurrences $N O\left[\boldsymbol{M}_{\boldsymbol{j}}\right]$.

Assumption 6: The product family comprises of several products $P_{N L}$.

Assumption 7: Each product $P_{p}$ is realized by a selected set of machines $\boldsymbol{P} \boldsymbol{M}_{\boldsymbol{p}}\left[\boldsymbol{M}_{\boldsymbol{j}}\right]$.

Assumption 8: Each product can use several duplications of machines $\boldsymbol{N} \boldsymbol{O}_{\boldsymbol{p}}\left[\boldsymbol{M}_{\boldsymbol{j}}\right]$.

In order to tackle the above two challenges, our problem is treated in two steps (sub-sections 3.2.1 and 3.2.2). Moreover, it represents a natural extension of to the works presented in Haddou Benderbal et al. (2017a and 2018).

More specifically, our problem is to determine the best layout of RMTs, while minimizing the penalties related to the non-satisfaction of machine-location association constraints in the system. The nonsatisfaction of these constraints may occur due to the conflict between different machines requirements. These penalties are based on the importance of each machine regarding the process plan as well as the product family.

\subsubsection{First-sub problem}

The formulation for the first sub-problem of our paper - i.e. logical environment that treats the transition effort evolution between products of the same product family - is adapted from Haddou Benderbal et al. (2017a). Thus, this sub-problem is based on the following concepts:

- Inclusion concept (i.e., inclusion of functionalities between machines). As depicted in Figure 1, It consists in choosing machines with richer functionalities compared to the needs of the initial product, thus offering the necessary functionalities to manufacture the initial product, but also other additional functionalities that can facilitate the evolution (the transition) towards other products from the same family. Therefore, the question that arises is to assess the relevance of replacing one machine (Mi) with another $(\mathrm{Mj})$ that includes it functionalities in all relevant process plans. It is a matter of finding the compromise between, on the one hand the need to minimize the effort of evolution between the products and on the other hand, the risks incurred related to the balance of the loads and the presence of alternative machines.

- Preserving the system's capacities in terms of: (i) the balance of loads and (ii) the presence of alternative machines. This is to take into consideration the risks involved with the inclusion concept of additional features. 
- Diversity of machines: It consists of selecting machines with partially similar capabilities in order to reduce exclusivity in the operations-machine relationships. This will reduce the risk of load imbalance and the risk of missing replacement machines.

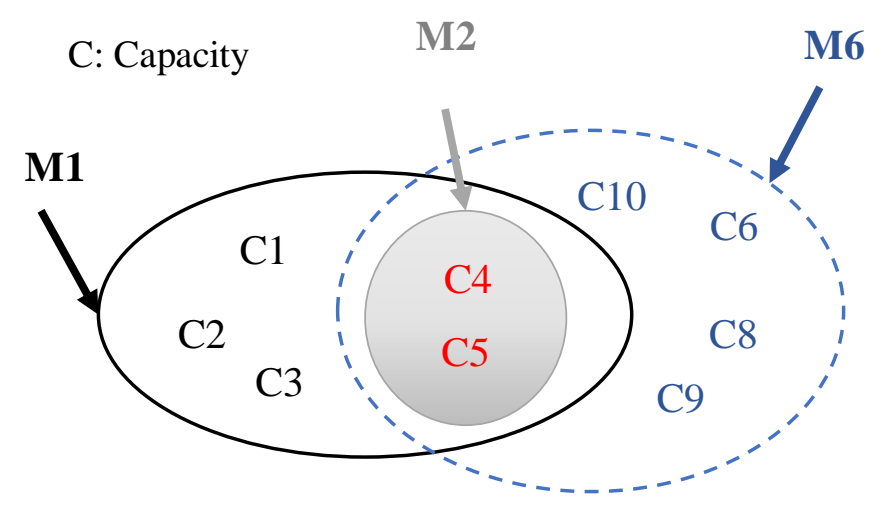

Fig. 1 Inclusion concept

Consequently, based on these concepts, our first sub-problem can be formulated as follows: (is guided by three criteria to optimize respectively:

1. The maximization of the average use of machines to reach equilibrium if possible, depicted by the average machine usage per product (AMUP) criterion as follows:

$$
\begin{gathered}
M U F_{p}\left(M_{j}\right)=\left(\frac{N O_{p}\left(M_{j}\right)}{O P T N_{p}}\right) \times \sum_{u=0}^{O P T N_{p}}\left(\alpha_{j}\left(O P_{u}^{p}\right) \times M_{j}\left(O P_{u}^{p}\right)\right) \\
\operatorname{Max}\left\{A M U P=\left(\frac{1}{N P \times N M f}\right) \sum_{j=1}^{N M f} \sum_{p=0}^{N P} M U F_{p}\left(M_{j}\right)\right\}
\end{gathered}
$$

where: $M_{j}\left(O P_{u}^{p}\right)=1$ if the machine $\mathrm{Mj}$ can accomplish operation u of product $\mathrm{Pp}, 0$ otherwise. $\alpha=1$ if the machine $\mathrm{Mj}$ is selected to perform operation $\mathrm{u}$ of product $\mathrm{Pp}, 0$ otherwise.

2. The maximization of the presence of replacement machines in the production lines of the product family formulated as follows.

$$
\operatorname{Max}\left\{M R P=\frac{1}{N P} \sum_{p=0}^{N P}\left(\frac{1}{O P T N_{p} \times N S M_{p}} \sum_{u=0}^{O P T N_{p}} \sum_{j=0}^{N S M_{p}}\left(\begin{array}{c}
M_{j}\left(O P_{u}^{p}\right) \\
\times N O_{p}\left(M_{j}\right)
\end{array}\right)\right)\right\}
$$

3. The minimization of the layout evolution effort (LEE). It quantifies the change that may occur to the selected machines and or to their rearrangement from one product to another among products of the family. The LEE is based on two aspects namely:

Machine similarities $\left(\mathbf{P S i m M}_{\mathbf{p}}\right)$ : It allows selecting the best machine layout in term of similarities between the selected machines. This later assesses the average similarity be- 
tween selected machines of products from the product family. The similarity calculation is based on the number and type of configuration changes between two given machines and it is formulated like so:

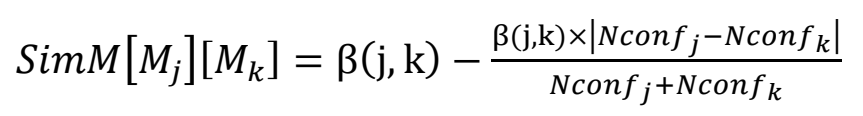

where:

$$
\beta(\mathrm{j}, \mathrm{k})=\left\{\begin{array}{l}
1, \text { if machines } M j \text { and } \mathrm{M} k \text { have the same basic modules } \\
0, \text { Otherwise } \\
k, j \in 1 . . N P M
\end{array}\right.
$$

In a second time, we determine the average similarity between machines, which are selected to realize a given product from the product family, thus:

$$
\operatorname{PSim}_{p}=\frac{1}{N S M_{p}} \sum_{j=1}^{N S M_{p}-1} \sum_{k=j+1}^{N S M_{p}} \operatorname{Sim} M\left[M_{j}\right]\left[M_{k}\right]
$$

Selected machine type difference (SMDifp): It concerns the difference between the selected machines types for each product. Here, it is necessary to determine the types of machines used for each product (SMT), the number of deleted machines (RM), and the number of machines that are added (AM), when changing from one product to another.

$$
\begin{aligned}
& R M=\sum_{j=0}^{N M} \operatorname{Max}\left(P M_{p}\left[M_{j}\right]-P M_{p^{\prime}}\left[M_{j}\right], 0\right) \\
& A M=\sum_{j=0}^{N M}\left|\operatorname{Min}\left(P M_{p}\left[M_{j}\right]-P M_{p^{\prime}}\left[M_{j}\right], 0\right)\right|
\end{aligned}
$$

The quantification of used type is calculated when moving between products of the same family as follows:

$$
S M T=\sum_{j=0}^{N M} \operatorname{Max}\left(P M_{p}\left[M_{j}\right], P M_{p^{\prime}}\left[M_{j}\right]\right)
$$

where:

$$
P M_{p}\left(M_{j}\right)= \begin{cases}1, & \text { if product } P p \text { is realized by machine } M_{j} \\ 0, & \text { otherwise }\end{cases}
$$


Using these three aspects namely SMT, AM and AR, we can formulate the difference between the selected machines types for each product as follows:

$$
S M D i f_{p}=\frac{A M+R M}{S M T}
$$

Based on these two aspects namely 'machine similarities' and 'selected machine type difference', the LEE is determined as follows:

$$
\operatorname{Min}\left\{L E E=\frac{1}{N P} \sum_{p=1}^{N P}\left(\frac{\operatorname{SMDif}_{p}}{\operatorname{PSimM}_{p}}\right)\right\}
$$

\subsubsection{Second-sub problem}

On the other hand, for our second sub-problem - i.e. physical environment that treats the machine layout problem - based on the numerous layout configurations of machines present in the literature, the generic model proposed in (Haddou Benderbal et al., 2018a) and depicted in Figure 2 is used. This later allows the representation of machine layouts configurations in the form of a matrix called localization matrix (LoC). LoC makes it possible to represent a layout configuration by specifying the distances between machines in the workshop according to the capacities and constraints of the system (ex. the constraints of size) as well as the available positions. This assumption makes our model more generic to include all the possible layout configurations. Note that, the distances between the positions (machines) rely on the transfer capacities between the machines and ensured by the chosen material handling system. They are provided by the decision maker and considered as input for our model. In Figure 2, L1, ..., L8 are the previously known candidate locations. They are intended to accommodate the selected machines to be part of the designed RMS, where the number of slots in the array corresponds to the total number of occurrences of the selected machines for the product family.

We expect to determine the best machines layout based on the following information:

(i) the characteristics and needs of the products to be considered from the product family,

(ii) the process plans to adopt to manufacture these products, and

(iii) the selected RMTs and the number of their respective occurrences, to be included in the new designed RMS.

For our case, a RMS is composed of several RMTs, having the capacity to manufacture several products of the same family. Each RMT has one or more instances to integrate into the workshop according to the needs and requirements of the process plan of each product. Note that, we consider a single unit of each product of the product family assigned to its optimal process plan to accomplish this product. Thus, the machines included in the design and their occurrences are known. Moreover, a machine can be used several times in any process plan and this number of uses may exceed the number of available occurrences 
for this machine (i.e. each machine can have multiple occurrences and appear several times in the process plan). Based on these inputs, it is possible to generate an initial layout using the given process plans of products from the product family.

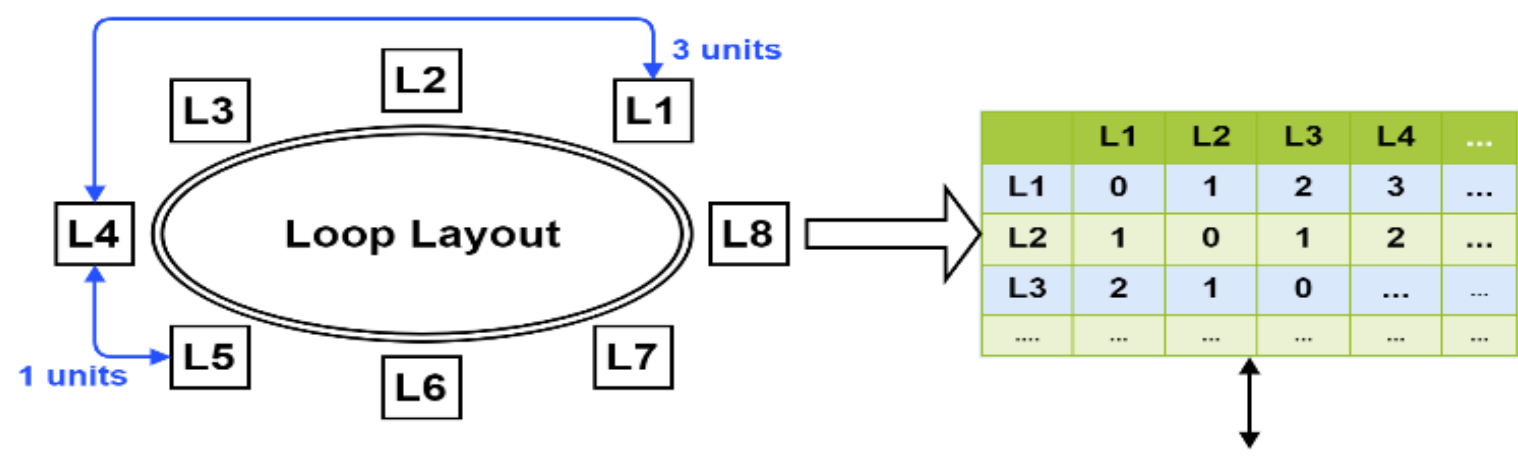

Open-field Layout

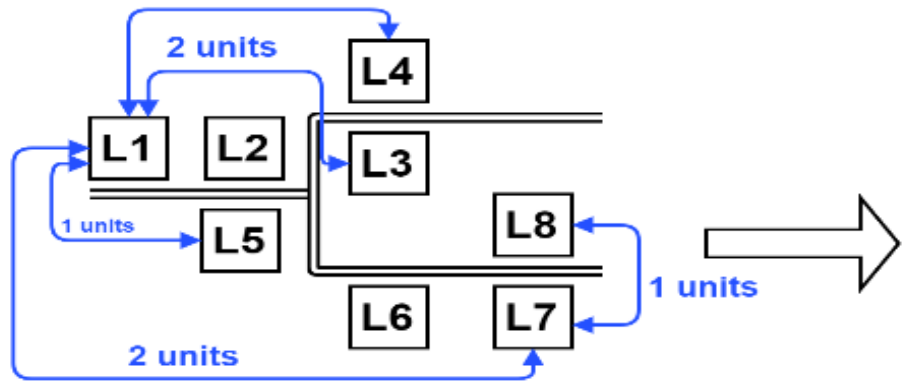

Location matrix

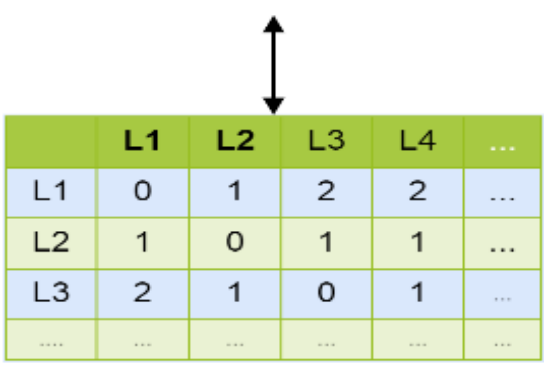

Fig. 2 Representation of layout configurations

The initial layout is generated according to the order of appearance of the machines in the adopted process plans as well as to the importance of these machines to the whole product family. For example, if a machine $M_{j}$ is used directly after a machine $M_{j^{\prime}}$, then the chosen layout must offer the possibility of a connection between the two machines. Hence, based on this initial layout as well as the constraints imposed by the adopted process plans, we define two new matrices respectively, maximum and minimum accepted distances (MaxAD, MinAD) between different machines and their occurrences as well. This set of information helps us to define the importance of each machine regarding the adopted process plans of all products from the product family.

The machine importance indicator $\operatorname{MI}\left(\mathrm{M}_{\mathrm{j}}\right)$ provides an insight over the dependency of different process plans of the product family regarding the selected machines. This later is adapted from the work of (Haddou Benderbal et al., 2018a) and formulated for all the given process plans of the product family as follows:

$$
M I\left(M_{j}\right)=\sum_{p=1}^{N P} M I_{p}\left(M_{j}\right) / N S M_{p}
$$

where: 


$$
\begin{gathered}
M I_{P}\left(M_{j}\right)=\sum_{k=1}^{N O_{p}\left[M_{j}\right]} \operatorname{MOI}_{p}\left(M_{j k}\right) \\
\operatorname{MOI}_{P}\left(M_{j o}\right)=C P_{P}\left(M_{j o}\right) \times \sum_{k=1}^{N S M_{P}^{o}} \frac{M R_{P}\left[M_{j o}\right]\left[M_{j^{\prime} k}\right]+M R_{P}\left[M_{j \prime k}\right]\left[M_{j o}\right]}{N O_{p}\left(M_{j}\right)}
\end{gathered}
$$

$C P_{P}(\mathrm{Mjo})$ : the number of occurrences of machine $\mathrm{Mj}$ in the adopted process plan of the product $\mathrm{Pp}$. $M R_{P}\left[M_{j o}\right]\left[M_{j k}\right]:$ the relation between all the occurrences of the machines used in the process plan of product Pp given by:

$M R_{P}\left[M_{j o}\right]\left[M_{j^{\prime} k}\right]=\left\{\begin{array}{r}1 \text { if the occurrence o of the machine } \mathrm{Mj} \text { is followed } \\ \text { by the occurrence } \mathrm{k} \text { of the machine } \mathrm{Mj}^{\prime} \\ 0 \text { otherwise }\end{array}\right.$

The importance index (Eq. 11) will help us later to determine the penalty that may occur if machine requirement in terms of position are not satisfied.

Once the machine importance metric is determined, for each occurrence of each machine for the whole products, it is possible to obtain an initial layout by the available inputs of our model. The initial assignment of machines to the available locations in the layout configuration is based on the sequence of these machines in the generated process plans as well as their importance indexes.

Using this initial layout, the required distance to respect between different selected machines for the whole needed products rom the product family is determined. Based on this generated initial layout and also the constraints imposed by the adopted process plans resulting from solving the first sub-problem, we can now define two new matrices respectively minimum accepted distance $\boldsymbol{M i n} \boldsymbol{A D}\left[\boldsymbol{M}_{\boldsymbol{j o}}\right]\left[\boldsymbol{M}_{\boldsymbol{j o}}^{\prime}\right]$ and maximum accepted distance $\boldsymbol{M a x} \boldsymbol{A D}\left[\boldsymbol{M}_{\boldsymbol{j o}}\right]\left[\boldsymbol{M}_{\boldsymbol{j o}}^{\prime}\right]$. These two matrices are generated for each process plan and calculated as follows:

The first step consists of identifying the occurrences retained in the initial layout and calculating the distance to the right $\left(D^{R}\right)$ and to the left $\left(D^{L}\right)$ between these occurrences. The following equations show the expressions of the right distance $D_{P \bar{I}}^{R_{k} k}$ and the left distance $D_{P \bar{I}}^{L_{-} k}$ between the occurrence of a machine $M_{j}$ at the position $P I$ and the position of an occurrence $k$ (of the same machine or another machine).

$$
D_{P I}^{R}{ }^{R}= \begin{cases}|k-P I| & \text { Si } k \leq P I \\ {[O P T N-k]+P I} & \text { Si } k>P I\end{cases}
$$




$$
D_{P \bar{I}}^{L_{-} k}= \begin{cases}|k-P I| & \text { Si } k \geq P I \\ {[O P T N-P I]+k} & \text { Si } k<P I\end{cases}
$$

where:

- PI: index of the occurrence retained by the initial layout in the process plan

- $k \in 1 . . O P T N$

To compute $\operatorname{MinAD}_{P}$ and $\mathrm{MaxAD}_{P}$, the distances between two given machines within the process plan of product $\mathrm{P}$, we consider all their respective occurrences in the process plan of the product $\mathrm{P}$ as follows:

$$
\begin{aligned}
& \operatorname{Min} A D_{P}\left[M_{j o}\right]\left[M_{j o}{ }^{\prime}\right]=\operatorname{Min}\left(D_{P I_{M_{j o}}}^{R_{M_{j o}}^{\prime}}, D_{P I_{M_{j o}}}^{L_{M_{j o}}^{\prime}}\right) \\
& \operatorname{Max}_{P}\left[D_{P o}\right]\left[M_{j o}{ }^{\prime}\right]=\operatorname{Min}\left(D_{P I_{M_{j o}}}^{R_{M_{j o}}^{\prime}}, D_{P I_{M_{j o}}}^{L_{M_{j o}}^{\prime}}\right)
\end{aligned}
$$

Based on this importance indicator, the penalty function which measures the level of satisfaction of the constraints of the different process plans by the generated layout of the RMTs can be computed. It quantifies to "what extent the locations chosen for the occurrences of the different machines respect the maximum and minimum distances?', while considering the importance of each one. Therefore, our problem is to minimize the penalty generated due to non-satisfaction of the constraints expressed by (Eq. 18):

$$
\operatorname{Min}\left\{\text { Penalité }=\sum_{j=1}^{N S M} \sum_{k=1}^{N S M} X_{j k} \times M I\left(M_{j}\right)\right\}
$$

where: $X_{j k}=\left\{\begin{array}{l}1, \text { if MinAD }\left[M_{j o}\right]\left[M_{j \prime k}\right] \leq \operatorname{LoC}\left[P\left(M_{j o}\right)\right]\left[P\left(M_{j \prime k}\right)\right] \leq \operatorname{Max} A D\left[M_{j o}\right]\left[M_{j \prime k}\right] \\ 0, \text { otherwise }\end{array}\right.$

\section{Proposed approach}

Hybrid algorithms are known to be very good search tools and displayed very satisfying results (Talbi, 2009). They are often used to solve conventional and real-world optimization problems. In this context, a two-phase based approach which combine the well know metaheuristic AMOSA and an exhaustivesearch based heuristic is proposed. In the first phase, from the multi-objective nature of our first subproblem, an adapted version of AMOSA is used (see Figure 3). The obtained results are considered, in the second phase, as inputs for the exhaustive-search based heuristic to solve the second sub-problem (i.e., machine layout problem of the selected machines in our product family). 


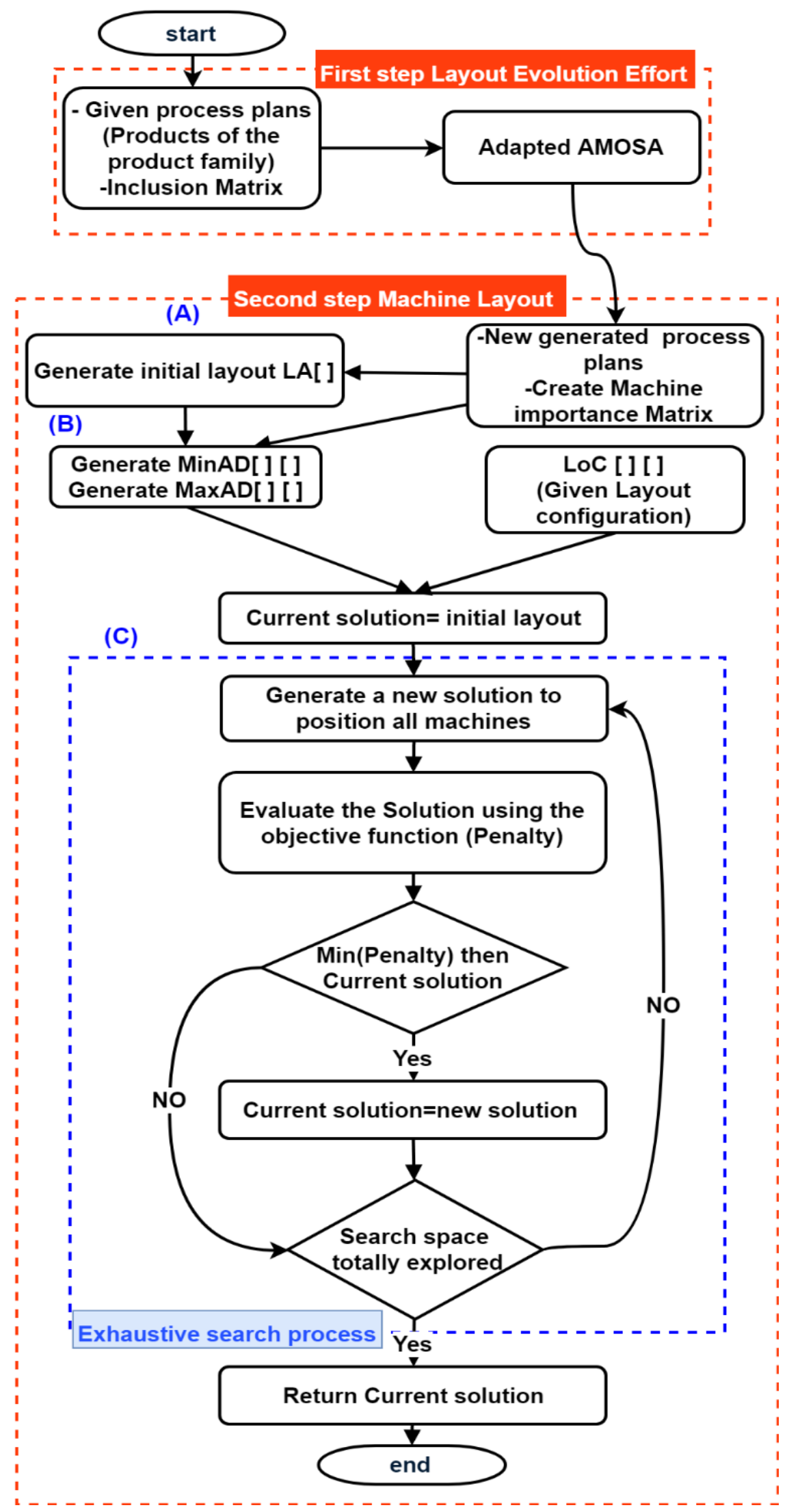

Fig. 3 Proposed Two-Phase based approach 


\section{$5 \quad$ Illustrative example and discussion}

In this section, digital experiments and analyzes are developed to show the applicability of our approach. A simple example of 3 products namely $\mathbf{P 1}, \mathbf{P 2}$ and $\mathbf{P 3}$ from the same family requiring respectively 8,8 and 7 different operations is used. Table 2, Table 3 and Table 4 detail respectively the adopted process plan of product P1, P2 and P3. The total number of different operations required for all the three products is $\mathrm{TFOP}=13$.

Table 3. Process plan of $\mathrm{P} 2$

\begin{tabular}{ccccccccc}
\hline Operation & OP1 & OP7 & OP6 & OP10 & OP2 & OP13 & OP11 & OP8 \\
Designated machines & M1 & M2 & M8 & M6 & M2 & M8 & M1 & M6
\end{tabular}

Table 4. Process plan of P3

\begin{tabular}{cccccccc}
\hline Operation & OP3 & OP5 & OP2 & OP12 & OP6 & OP4 & OP7 \\
\hline Designated machines & M9 & M6 & M1 & M8 & M1 & M8 & M2 \\
\hline
\end{tabular}

Table 5 represents the inclusion matrix (based on the inclusion concept) with the set of all initial 10 candidates machines (the same set of machines used to generate initial process plans of P1, P2 and P3). AMOSA perturbation phase is based on this matrix (i.e., AMOSA is used in the first phase). This first step is simple. In fact, based on the inclusion concept, we observe for the machines in our solution set, if there is a common machine which include their capabilities i.e. can these machines be replaced in terms of fulfilling the requirements of the tasks (originally assigned to the machine to be replaced).

Table 5. Machine inclusion matrix

\begin{tabular}{lcccccccccc} 
& M1 & M2 & M3 & M4 & M5 & M6 & M7 & M8 & M9 & M10 \\
\hline M1 & 1 & & & & & & & & & \\
M2 & 1 & 1 & & & 1 & 1 & 1 & & & \\
M3 & & & 1 & & & & 1 & & & \\
\hline M4 & 1 & & & 1 & 1 & & 1 & 1 & 1 & \\
\hline M5 & & & & 1 & & & & & \\
\hline M6 & & & & & 1 & & & \\
\hline M7 & & & & 1 & & & 1 & & \\
\hline M8 & 1 & & & & & & 1 & & 1 & \\
\hline M9 & & & & 1 & 1 & 1 & & 1 & 1 \\
\hline M10 & & & & & & & & & & \\
\hline
\end{tabular}


Table 5 explores from left to right row by row as follows: 1 in a cell ij indicates that machine Mi is included in the machine of column Mj. e.g. cell 2-5 $\Rightarrow$ the capacities of machine M2 are included in M5, this means that M5 can realize all the requested operations that M2 can do.

Table 6 presents a randomly generated configuration layout. We note that, the number of available candidate locations in the shop floor is greater than or equal to the total number of all occurrences of selected machines in the process plan. It represents the maximum number of all occurrences in a process plan of the product family. For the sake of simplicity, our example considers 7 candidate machines positions for process plan of $\mathbf{P 1}$.

Table 6. Input location matrix (Layout configuration)

\begin{tabular}{cccccccc}
\hline & L1 & L2 & L3 & L4 & L5 & L6 & L7 \\
\hline L1 & 0 & 3 & 2 & 1 & 5 & 4 & 8 \\
\hline L2 & 3 & 0 & 2 & 4 & 6 & 6 & 9 \\
\hline L3 & 2 & 2 & 0 & 1 & 4 & 3 & 7 \\
\hline L4 & 1 & 4 & 1 & 0 & 5 & 1 & 4 \\
\hline L5 & 5 & 6 & 4 & 5 & 0 & 8 & 4 \\
\hline L6 & 4 & 6 & 3 & 1 & 8 & 0 & 2 \\
\hline L7 & 8 & 9 & 7 & 4 & 4 & 2 & 0 \\
\hline
\end{tabular}

Table 7 represents the input solution used by AMOSA to get the best transition (guided by the three objectives AMUP, MRP and LEE). The evaluation of this input in terms of these three objectives is shown in Table 8.

Table 7. AMOSA input solution

\begin{tabular}{|lllllll} 
PP (P1) & M1 & M2 & M3 & M4 & M6 & M10 \\
\hline PP (P2) & M1 & M2 & M6 & M8 & M9 & - \\
\hline PP (P3) & M1 & M2 & M6 & M8 & M9 & - \\
\hline
\end{tabular}

Table 8. Evaluation of input solution

\begin{tabular}{ccc}
\hline AMUP & MRP & LEE \\
\hline 0.133 & 0.726 & 6.637 \\
\hline
\end{tabular}


The results of the first phase as well as the evaluation of this solution are shown respectively in Table 9 and Table 10. We can see that, it succeeds to reduce the evolution effort by using the inclusion concept and replacing original machines while slightly increasing the presence of alternative machines as well as the average use of machines among all operations of the product family.

Table 9. AMOSA output solution

\begin{tabular}{|lllllll}
\hline PP (P1) & M1 & M7 & M7 & M7 & M1 & M10 \\
\hline PP (P2) & M1 & M7 & M1 & M1 & M9 & - \\
\hline PP (P3) & M1 & M7 & M1 & M1 & M9 & - \\
\hline
\end{tabular}

Table 10. Evaluation of output solution

\begin{tabular}{ccc}
\hline AMUP & MRP & LEE \\
\hline 0.271 & 0.792 & 4.80 \\
\hline
\end{tabular}

To help the reader understating the explanation of the second phase, we will limit our discussion to just one process plan (for product P1) and see the impact when using the transition effort (first phase) and without the use of this latter. The second phase begins by generating the initial layout as depicted in Table 11. This initial layout is based on the importance indicator of each machine as well as the process plans of products from the product family. In Table 11, the notation M1_1 and M1_2 means respectively, occurrence 1 of machine M1 and occurrence 2 of machine M1.

Table 11. Initial layout without using first phase

\begin{tabular}{lccccccc}
\hline Candidate location & L1 & L2 & L3 & L4 & L5 & L6 & L7 \\
\hline Machine occurrence & M2 & M10 & M1_1 & M4 & M6 & M1_2 & M3
\end{tabular}

Table 12 and Table 13 represent respectively the generated MinAD and MaxAD matrices for the different selected machines (for product P1) without using the first step. These two tables are used to determine the penalty of the generated layout and to which extent it respect the constraints imposed by these two tables.

Table 14 depicts the results of machine layout for the process plan of product P1 without using the results from the first phase of our approach. The model tests all possible layouts. Hence, in this table, only the best and worst solutions according to penalty (from 100 first results) are shown. We can see that the smallest change can lead to a significant change in the incurred penalty. 
Table 12. MinAD without using first phase

\begin{tabular}{cccccccc}
\hline & M1_1 & M1_2 & M2 & M3 & M4 & M6 & M10 \\
\hline M1_1 & 0 & 4 & 2 & 3 & 2 & 5 & 1 \\
\hline M1_2 & 4 & 0 & 2 & 1 & 2 & 1 & 3 \\
\hline M2 & 2 & 2 & 0 & 1 & 4 & 3 & 1 \\
\hline M3 & 3 & 1 & 1 & 0 & 3 & 2 & 2 \\
\hline M4 & 2 & 2 & 4 & 3 & 0 & 1 & 1 \\
\hline M6 & 5 & 1 & 3 & 2 & 1 & 0 & 2 \\
\hline M10 & 1 & 3 & 1 & 2 & 1 & 2 & 0 \\
\hline
\end{tabular}

Table 13. MaxAD without using first phase

\begin{tabular}{cccccccc}
\hline & M1_1 & M1_2 & M2 & M3 & M4 & M6 & M10 \\
\hline M1_1 & 0 & 4 & 6 & 5 & 6 & 5 & 7 \\
\hline M1_2 & 4 & 0 & 6 & 7 & 6 & 7 & 5 \\
\hline M2 & 6 & 6 & 0 & 7 & 4 & 5 & 7 \\
\hline M3 & 5 & 7 & 7 & 0 & 5 & 6 & 6 \\
\hline M4 & 6 & 6 & 4 & 5 & 0 & 7 & 7 \\
\hline M6 & 5 & 7 & 5 & 6 & 7 & 0 & 6 \\
\hline M10 & 7 & 5 & 7 & 6 & 7 & 6 & 0 \\
\hline
\end{tabular}

Table 14. Resulted layouts without using first phase

\begin{tabular}{lcccccccc} 
Candidate position & L1 & L2 & L3 & L4 & L5 & L6 & L7 & Penalty \\
\hline Best solution & M6 & M3 & M10 & M4 & M1_2 & M2 & M1_1 & 10 \\
\hline Worst solution & M4 & M3 & M10 & M6 & M1_1 & M1_2 & M2 & 20
\end{tabular}

From the above panel of experiments, when considering the transition effort (first and second phases), the following observations can be delivered:

Observation 1: the results of the first phase have reduced the number of machines to be placed (i.e., from Table 9, we can see 3 instead of 6 machines for the process plan of P1). 
Observation 2. this leads to the reduction of the number of constraints imposed by the process plans and gives more freedom to place the machines on the shop floor.

Observation 3: this relaxation has made the placement of machines easier.

Consequently, as depicted in Table 15, eliminating the penalty for this specific example as best. Nevertheless, the relaxation comes with a considerable dependency of process plans on small set of machines, even with the consideration of replacement machines amongst each other. One of the limitations of our approach is mainly related to computational time when considering more than 12 machines due to the exhaustive search method. Furthermore, one of the natural extensions of our current work is to consider these two problems to enhance the results of our approach.

Table 15. Resulted layouts using both phases (1 and 2)

\begin{tabular}{lcccccccc}
\hline Candidate position & L1 & L2 & L3 & L4 & L5 & L6 & L7 & Penalty \\
\hline Best solution & M7 & M10 & M1 & - & - & - & - & 0 \\
\hline Worst solution & M7 & - & M10 & M1 & - & - & - & 21.34 \\
\hline
\end{tabular}

\section{Conclusion and future work directions}

Nowadays, it is well known that RMS is one of the most promising and attractive research topics. This paper studied the relationships that link the RMS to its both logical and physical environments. From one hand, by considering the evolution of a product within the same product family for which the RMS is designed. And, by considering the machine layout problem in RMS. Moreover, due to the lack of research works dealing with the machine layout problem for RMS, we developed a new hybrid approach combining an adapted version of the well-known AMOS and an exhaustive search-based heuristic. This study is based on the premise that a RMS is designed to make one type of product, but must encompass the features that allow it to evolve to make other products from the same family. Finally, the presented illustrative numerical example showed the applicability of the developed approach.

For future work, we aim to integrate both the logical and physical environments issues with the process plan generation, to study the impact of product evolution and the layout on the design RMSs. Furthermore, to counter the computational time limitation imposed by the exhaustive search method used in phase two, the use other evolutionary algorithms such as NSGA-II and bees algorithms is an interesting research direction. 


\section{References}

Altuntas S. and Selim H. (2012). Facility layout using weighted association rule-based data mining algorithms: Evaluation with simulation. Expert Systems with Applications, 39(1), 3-13.

Andersen A.L., Brunoe T.D., Nielsen K. and Rösiö C. (2017). Towards a generic design method for reconfigurable manufacturing systems: analysis and synthesis of current design methods and evaluation of supportive tools. Journal of Manufacturing Systems, 42:179-195.

Andersen A.L., Brunoe T. D. and Nielsen K. (2015). Reconfigurable manufacturing on multiple levels: literature review and research directions. In IFIP International Conference on Advances in Production Management Systems (pp. 266-273). Springer.

Ashraf M. and Hasan F. (2016). Product family formation for RMS - a review. In proceedings of the NCMEI3, Aligarh Muslim University, Aligarh, India.

Azevedo M.M., Crispim J.A. and de Sousa J.P. (2017). A dynamic multi-objective approach for the reconfigurable multi-facility layout problem. Journal of Manufacturing Systems, 42:140-52.

Bandyopadhyay S., Saha S., Maulik U. and Deb K. (2008). A simulated annealing-based multiobjective optimization algorithm: AMOSA. IEEE transactions on evolutionary computation, 12(3), 269-283.

Battaïa O., Dolgui A. and Guschinsky N. (2017). Decision support for design of reconfigurable rotary machining systems for family part production. International Journal of Production Research, 55, $1368-1385$.

Benjaafar S., Heragu S. S. and Irani S. A. (2002). Next generation factory layouts: research challenges and recent progress. Interfaces, 32(6), 58-76.

Bi Z. M., Lang S. Y., Shen W. and Wang L. (2008). Reconfigurable manufacturing systems: the state of the art. International Journal of Production Research, 46, 967-992.

Bortolini M., Galizia F.G. and Mora C. (2018). Reconfigurable manufacturing systems: Literature review and research trend. Journal of manufacturing systems, 49, 93-106.

Devise O. and Pierreval H. (2000). Indicators for measuring performances of morphology and material handling systems in flexible manufacturing systems. International Journal of Prod Economics, 64(1), 209-218.

Drira A., Pierreval H. and Hajri-Gabouj S. (2007). Facility layout problems: A survey. Annual Reviews in Control, 31(2), 255-267.

Dou J., Dai X. and Meng Z. (2010). Optimisation for multi-part flow-line configuration of reconfigurable manufacturing systems using GA. International Journal of Production Research, 48(14): 4071-4100.

ElMaraghy H. A. (2007). Reconfigurable process plans for responsive manufacturing systems. In Digital enterprise technology (pp. 35-44). Springer US. 
Gadalla M. and Xue D. (2018). An approach to identify the optimal configurations and reconfiguration processes for design of reconfigurable machine tools. International Journal of Production Research, 56:11, pages 3880-3900.

Galan R., Racero J., Eguia I. and Garcia J.M. (2007). A systematic approach for product families formation in reconfigurable manufacturing systems. Robot Computer Integrated Manufacturing, 23(5):489-502.

Goyal K. K. and Jain P. K. (2016). Design of reconfigurable flow lines using MOPSO and maximum deviation theory. The International Journal of Advanced Manufacturing Technology, 84 (5-8): 15871600.

Goyal K.K., Jain P.K. and Jain M. (2013). A comprehensive approach to operation sequence similarity based part family formation in the reconfigurable manufacturing system. International Journal of Production Research, 51(6):1762-1776.

Guan X., Dai X., Qiu B. and Li J. (2012). A revised electromagnetism-like mechanism for layout design of reconfigurable manufacturing system. Computer and Industrial Engineering, 63(1): 98-108.

Haddou Benderbal H., Dahane M. and Benyoucef L. (2017a). Flexibility based multi-objective approach for machines selection in reconfigurable manufacturing system (RMS) design under unavailability constraints. International Journal of Production Research, 55(20): 6033-6051

Haddou Benderbal H., Dahane M. and Benyoucef L. (2017b). Layout evolution effort for product family in Reconfigurable Manufacturing System design, In IFAC-PapersOnLine, Vol. 50, Issue 1, Pages 10166-10171, ISSN 2405-8963

Haddou Benderbal H., Dahane M. and Benyoucef L. (2018a). Exhaustive Search Based Heuristic for Solving Machine Layout Problem in Reconfigurable Manufacturing System Design, In IFACPapers, Volume 51, Issue 11, Pages 78-83, ISSN 2405-8963.

Haddou Benderbal, H., Dahane, M. and Benyoucef, L. (2018b). Modularity assessment in reconfigurable manufacturing system (RMS) design: an Archived Multi-Objective Simulated Annealing-based approach. The International Journal of Advanced Manufacturing Technology (2018) 94: 729.

Heragu S., Zijm W. H. M., Meng G., Heragu S. S., van Ommeren J. C. W. and van Houtum G-J. (2001). Design and analysis of reconfigurable layout systems. (Memorandum faculteit TW; No. 1604). Enschede: Stochastic Operations Research (SOR).

Huang L., Gao Y., Qian F., Tang S. and Wang D. (2011). Configuration selection for reconfigurable manufacturing systems by means of characteristic state space. Chin J Mech Eng, 24(1):23.

Kashkoush M. and ElMaraghy H. (2014). Product Family Formation for Reconfigurable Assembly Systems. Procedia CIRP, Vol. 17, 302-307. 
Koren Y. (2010). The global manufacturing revolution: product-process-business integration and reconfigurable systems (Vol. 80). John Wiley \& Sons.

Koren, Y. and Shpitalni, M. (2010). Design of reconfigurable manufacturing systems. Journal of Manufacturing Systems, 29(4), 130-141.

Koren Y., Wang W. and Gu X. (2017). Value creation through design for scalability of reconfigurable manufacturing systems. International Journal of Production Research, 55(5), 1227-1242.

Koren Y., Gu X. and Guo W. (2018a). Reconfigurable manufacturing systems: Principles, design, and future trends. Frontiers of Mechanical Engineering, Vol. 13, N 2, 121-136.

Koren Y., Gu X. and Guo W. (2018b). Choosing the system configuration for high-volume manufacturing. International Journal of Production Research, 56(1-2), 476-490.

Maganha I., Silva C. and Ferreira, L. M. D. (2018). Understanding reconfigurability of manufacturing systems: An empirical analysis. Journal of Manufacturing Systems, 48, 120-130.

Maniraj M, Pakkirisamy V. and Parthiban P. (2014). Optimisation of process plans in re- configurable manufacturing systems using ant colony technique. International Journal of Enterprise Network Management, 6(2):125-38.

Musharavati F. and Hamouda A.M.S. (2012). Enhanced simulated-annealing-based algorithms and their applications to process planning in reconfigurable manufacturing systems. Advanced Engineering Software, 45(1):80-90.

Musharavati F. and Hamouda A.M.S. (2012). Simulated annealing with auxiliary knowledge for process planning optimization in reconfigurable manufacturing. Robot and Computer Integrated Manufacturing, 28(2):113-31.

Nallakumarasamy G., Srinivasan P. S. S., Raja K. V. and Malayalamurthi R. (2011). Optimization of operation sequencing in CAPP using superhybrid genetic algorithms-simulated annealing technique. ISRN Mechanical Engineering, 2011.

Pattanaik L.N. and Kumar V. (2011). Product family formation for reconfigurable manufacturing using a bi-criterion evolutionary algorithm. The International Journal of Industrial Engineering: Theory, Applications and Practice 18(9): 493-505.

Rehman A.U. and Babu A. S. (2013). Reconfigurations of manufacturing systems — an empirical study on concepts, research, and applications. The International Journal of Advanced Manufacturing Technology, 66, 107-124.

Renzi C., Leali F., Cavazzuti M. and Andrisano A. (2014). A review on artificial intelligence applications to the optimal design of dedicated and reconfigurable manufacturing systems. The International Journal of Advanced Manufacturing Technology, 72, 403-418. 
Saxena L.K. and Jain P.K. (2012). A model and optimisation approach for reconfigurable manufacturing system configuration design. International Journal of Production Research, 50(12):3359-3381.

Shabaka, A. and ElMaraghy H. A. (2007). Generation of machine configurations based on product features. International Journal of Computer Integrated Manufacturing, 20, 355-369.

Sharma P., and Singhal S. (2016). Design and evaluation of layout alternatives to enhance the performance of industry. OPSEARCH, 53(4), 741-760.

Singh S. P. and Sharma,R. R. (2006). A review of different approaches to the facility layout problems. The International Journal of Advanced Manufacturing Technology, 30(5-6), 425-433.

Talbi E. G. (2009). Metaheuristics: from design to implementation. John Wiley \& Sons (Vol. 74).

Touzout F. A. and Benyoucef L. (2018a). Multi-objective sustainable process plan generation in a reconfigurable manufacturing environment: exact and adapted evolutionary approaches. International Journal of Production Research, (pp. 1-17).

Touzout F. A. and Benyoucef L. (2018b). Sustainable multi-unit process plan generation in a reconfigurable manufacturing environment: A comparative study of three hybrid-meta-heuristics. In 23rd IEEE International Conference on Emerging Technologies and Factory Automation (ETFA) (pp. 661-668). IEEE volume 1.

Wang G.X., Huang S.H., Shang X.W., Yan Y. and Du J.J. (2016). Formation of part family for reconfigurable manufacturing systems considering bypassing moves and idle machines. Journal of

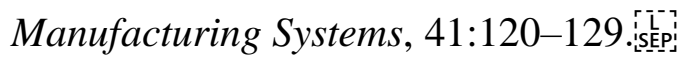

